\title{
Predictors of Lymph Node Involvement by Soft Tissue Sarcoma of the Trunk and Extremity: An Analysis of the National Cancer Database
}

Joseph A. Miccio ${ }^{1}$, Vikram Jairam ${ }^{1}$, Sarah Gao ${ }^{1}$, Alexander Augustyn ${ }^{2}$, Oluwadamilola T. Oladeru ${ }^{3}$, Benjamin E. Onderdonk ${ }^{4}$, Mudit Chowdhary ${ }^{5}$, Dale Han ${ }^{6}$, Sajid Khan ${ }^{7,8}$, , Gary Friedlaender ${ }^{9}$, Dieter M. Lindskog ${ }^{9}$, Hari A. Desphande ${ }^{10}$, Heather Osborn ${ }^{11}$, Kenneth B. Roberts ${ }^{12}$, Kirtesh R. Patel ${ }^{1}$

1. Department of Therapeutic Radiology, Yale School of Medicine, New Haven, USA 2. Department of Radiation Oncology, The University of Texas MD Anderson Cancer Center, Houston, USA 3. Department of Radiation Oncology, Massachusetts General Hospital/Harvard Medical School, Boston, USA 4. Department of Radiation and Cellular Oncology, University of Chicago Medical Center, Chicago, USA 5. Department of Radiation Oncology, Rush University Medical Center, Chicago, USA 6. Department of Surgical Oncology, Oregon Health and Science University, Portland, USA 7. Department of Surgery, Yale School of Medicine, New Haven, USA 8. Department of Surgical Oncology, Yale School of Medicine, New Haven, USA 9. Department of Orthopaedics and Rehabilitation, Yale School of Medicine, New Haven, USA 10. Department of Medical Oncology, Yale School of Medicine, New Haven, USA 11. Department of Otolaryngology, Yale School of Medicine, New Haven, USA 12. Department of Radiation Oncology, Yale School of Medicine, New Haven, USA

Corresponding author: Joseph A. Miccio, joseph.miccio@yale.edu

\section{Abstract}

Background and Objectives

Lymph node metastases (LNM) in soft tissue sarcoma (STS) of the trunk and extremity are rare but are associated with worse survival. Established risk factors for LNM in this group are based on small institutional retrospective reviews. This study identifies the risk factors associated with LNM in otherwise non-metastatic trunk/extremity STS patients using the National Cancer Database (NCDB) and sought out to delineate a high-risk group that may be considered for pathologic nodal evaluation.

\section{Methods}

The files of 10,731 patients with STS of the trunk/extremity without distant metastasis from 2004 - 2015 were evaluated. Exclusion criteria included neoadjuvant therapy and a lack of pathologic nodal evaluation. Univariate and multivariable logistic regression models were performed to evaluate variables associated with LNM.

Results

Received 08/06/2019

Review began 08/16/2019 Review ended 10/24/2019 Published 10/30/2019

๑) Copyright 2019

Miccio et al. This is an open access article distributed under the terms of the Creative Commons Attribution License CC-BY 3.0., which permits unrestricted use, distribution, and reproduction in any medium, provided the original author and source are credited.
Of the total of 10,731 patients, 223 (2.1\%) had LNM. On multivariable analysis, LNM was associated with Grade 3 tumors (odds ratio (OR) 15.6, 95\% confidence interval (CI) $6.36-38.04, \mathrm{p}<0.001$ ) and clear cell/angiosarcoma/rhabdomyosarcoma/epithelioid (CARE) histology (OR 4.72, 95\% CI 3.35 - 6.66, p < 0.001), lymphovascular invasion (LVI) (OR 5.86, 95\% CI $3.33-10.31, \mathrm{p}<0.001$, and bone invasion (BI) (OR 2.73, 95\% CI $1.32-5.61, p=0.006)$. Patients with Grade 3 CARE tumors $(n=402)$ had an $11.9 \%$ risk of LNM vs. $1.7 \%$ of adults without all these characteristics $(\mathrm{p}<0.001)$. Patients with Grade 3 CARE tumors and either LVI or BI $(n=36)$ had a $33.3 \%$ risk of LNM.

Conclusions

High-grade and CARE histology are associated with LNM in STS. Adult patients with both features have an overall $11.9 \%$ risk of LNM and may be considered for pathologic LN assessment, particularly with the presence of LVI or BI.

Categories: Oncology

Keywords: soft tissue sarcoma, trunk, extremity, lymph node metastasis, national cancer database (ncdb)

\section{Introduction}

Lymph node metastases (LNM) in adults with trunk/extremity soft tissue sarcoma (STS) are rare, generally affecting $<5 \%$ of STS patients [1-3]. However, patients with lymph node involvement have worse overall survival. Several retrospective studies have shown that patients with LNM metastasis have poorer survival [3-4], and the American Joint Commission on Cancer (AJCC) has changed the group staging for lymph nodepositive patients with trunk/extremity sarcoma from Stage III to Stage IV. Furthermore, retrospective 
evidence suggests that lymphadenectomy in patients with LNM from STS is necessary for long-term survival $[1-3,5-7]$. Thus, it is important to identify patients with STS at high risk for LNM, as these patients may be candidates for pathologic lymph node assessment.

Previous studies defining risk factors for nodal metastasis are primarily based on small, single-institution retrospective studies $[1,3,7-8]$. These series have reported an incidence of lymph node metastasis of $2 \%$ $7 \%$. Reports have also identified several variables associated with the development of lymph node metastasis, including histologic subtype, tumor grade [1, 8-9], patient age [2, 8], and primary tumor size [7-8].

The aim of this study is to identify risk factors associated with lymph node metastasis in STS patients without distant metastasis using the National Cancer Database (NCDB). We also aimed to identify a clinically relevant high-risk group with an increased risk of harboring disease in regional lymph nodes that may benefit from pathologic LN (lymph node) assessment.

\section{Materials And Methods}

The NCDB is a joint project of the Commission on Cancer $(\mathrm{CoC})$ of the American College of Surgeons and the American Cancer Society, which consists of de-identified information regarding patient demographics, tumor characteristics, first-course treatment for the corresponding diagnosis, and survival for approximately $70 \%$ of the United States (US) population [10]. All pertinent cases are reported regularly from CoC-accredited centers and compiled into a unified dataset, which is then validated. The NCDB contains information not included in the Surveillance, Epidemiology, and End Results (SEER) database, including details regarding the use of systemic therapy. The data used in the study were derived from a de-identified NCDB file (2004 - 2015). The American College of Surgeons and the CoC have not verified and are neither responsible for the analytic or statistical methodology employed, nor the conclusions drawn from these data by the investigators. As all patient information in the NCDB database is de-identified, this study was exempt from institutional review board evaluation.

The 2015 NCDB Participant User File contains 96,522 patients with soft tissue sarcoma diagnosed between 2004 and 2015. We included only patients with primary site codes of C471, C472, C476, C478, C479, C491, C492, C496, C498, and C499, corresponding to the ICD-O-3 codes included for trunk/extremity STS via the American Joint Committee on Cancer (AJCC), 8th edition $(n=55,469)[11]$. Other exclusion criteria included distant metastatic disease $(n=8,979)$, lack of pathologic nodal evaluation $(n=29.684)$, unknown or discrepant grade $(n=3,231)$, discordant nodal status and stage group $(n=7)$, and any patients receiving neoadjuvant therapy or if receipt of neoadjuvant therapy was unknown $(n=2,837)$. The remaining patients were categorized as either having a pathologically node-positive or node-negative status. Figure 1 shows the Consolidated Standards of Reporting Trials (CONSORT) diagram for the study [12]. Our final cohort consisted of 10,731 patients, 223 of whom had pathologic lymph node involvement. 


\section{Cureus}

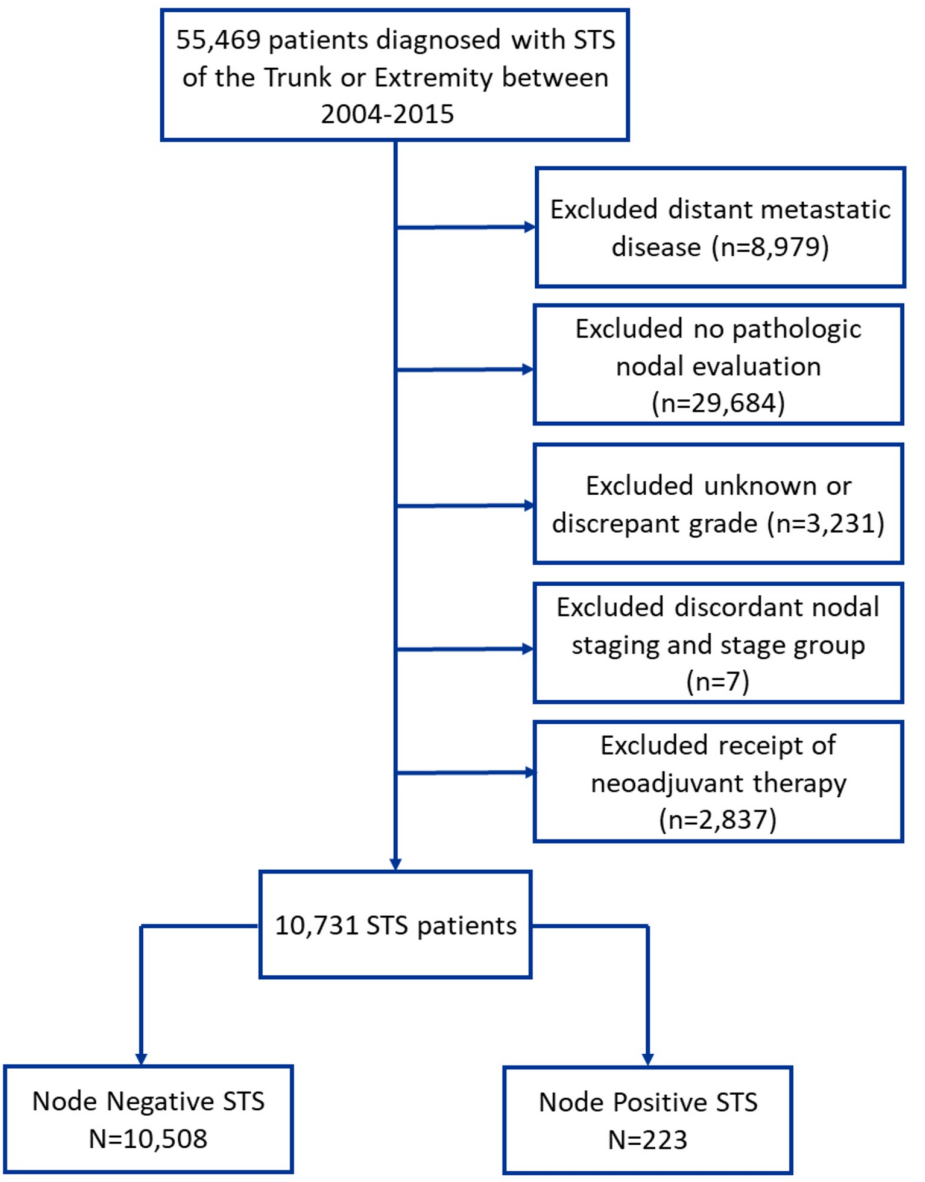

\section{FIGURE 1: Consolidated standards of reporting trials diagram delineating cohort selection}

The final cohort consisted of 10,508 trunk/extremity soft tissue sarcoma patients without lymph node metastasis $(97.9 \%)$ and 223 with lymph node metastasis $(2.1 \%)$.

STS: soft tissue sarcoma

The following disease-related variables were evaluated for association with LN involvement: grade, histology, tumor size, tumor location (trunk vs. extremity), LVI, BI, and neurovascular invasion (NVI). We also included the demographic factors of age, sex, and race. Age was evaluated both as a continuous variable and categorical variable (< 18 and $\geqslant 18)$. The histologies of clear cell, angiosarcoma, rhabdomyosarcoma, and epithelioid (CARE) were grouped as "CARE" histologies, given their higher propensity for LNM as previously described $[4,13]$ Other categories for histology included synovial sarcoma, given conflicting reports regarding its association with LNM [14-16], and "other." Univariate comparisons between the LNM cohort and node-negative cohort were made via the Chi-square test. Univariate and logistic regression models were performed to evaluate variables associated with pathologic node positivity. Variables associated with LNM with a P-value $<0.1$ were included in the multivariable logistic regression model using a forward stepwise method, as were variables that were previously shown to have an effect on LNM, such as tumor size [7-8]. Odds ratios and $95 \%$ confidence intervals were generated. P-values were derived from two-tailed tests. The analysis was performed using STATA, version 13 (StataCorp LLC, College Station, TX) and P-values $<0.05$ were considered statistically significant.

\section{Results}

Ten thousand seven hundred and thirty-one soft tissue sarcoma patients meeting the inclusion criteria were identified which included 10,508 patients without LNM (97.9\%) and 223 patients with LNM (2.1\%). The cohort characteristics are detailed in Table 1 . The LNM cohort had a higher proportion of pediatric cases (16, $7.2 \%$ ) compared with the node-negative group ( 235 cases, $2.2 \%, \mathrm{p}<0.001$ ), but race was balanced between the node-positive and node-negative cohorts $(\mathrm{p}=0.449)$. The LNM cohort, compared to the node-negative group, had a higher proportion of primary tumors that were in the trunk (47 cases, $21.1 \%$ vs. 1,433 cases, $13.6 \%$, p < 0.001 ), Grade 3 (198 cases, $88.8 \%$ vs. 5,929 cases, $56.4 \%, \mathrm{p}<0.001$ ), CARE histology (56 cases, $25.1 \%$ vs 458 cases, $4.4 \%, \mathrm{p}<0.001$ ), LVI ( 26 cases, $11.7 \%$ vs. 199 cases, $1.9 \%, \mathrm{p}<0.001$ ), NVI ( 7 cases, $3.1 \%$ 


\section{Cureus}

vs. 113 cases, $1.1 \%, \mathrm{p}=0.008)$, and BI (10 cases, $4.5 \%$ vs. 105 cases, $1.0 \%, \mathrm{p}<0.001)$. The CARE histology category contained 61 (11.9\%) clear cell cases, 180 (35.0\%) angiosarcoma cases, 139 (27.0\%)

rhabdomyosarcoma cases, and 134 (26.1\%) epithelioid sarcoma cases, and there were 446 cases of synovial sarcoma.

\begin{tabular}{|c|c|c|c|c|c|}
\hline Variable & Node-negative & $\%$ & Node-positive & $\%$ & P-value \\
\hline Total & 10,508 & 97.9 & 223 & 2.1 & \\
\hline Age (years) & & & & & $<0.001$ \\
\hline$<18$ & 235 & 2.2 & 16 & 7.2 & \\
\hline$\geq 18$ & 10,237 & 97.8 & 207 & 92.8 & \\
\hline Sex & & & & & 0.999 \\
\hline Male & 5,654 & 53.8 & 120 & 53.8 & \\
\hline Female & 4,854 & 46.2 & 103 & 46.2 & \\
\hline Race & & & & & 0.449 \\
\hline White & 8,743 & 83.2 & 183 & 82.1 & \\
\hline Black & 1,192 & 11.3 & 20 & 13.5 & \\
\hline Asian & 283 & 2.7 & 7 & 3.1 & \\
\hline Unknown & 290 & 2.8 & 3 & 1.4 & \\
\hline Location & & & & & 0.001 \\
\hline Trunk & 1,433 & 13.6 & 47 & 21.1 & \\
\hline Extremity & 9,076 & 86.4 & 176 & 78.9 & \\
\hline Size & & & & & $<0.001$ \\
\hline$\leq 5 \mathrm{~cm}$ & 9.926 & 94.5 & 195 & 87.4 & \\
\hline$>5 \mathrm{~cm}$ & 52 & 0.5 & 4 & 1.8 & \\
\hline Unknown & 530 & 5.0 & 24 & 10.8 & \\
\hline Grade & & & & & $<0.001$ \\
\hline 1 & 2,850 & 27.1 & 5 & 2.2 & \\
\hline II & 1,729 & 16.5 & 20 & 9.0 & \\
\hline III & 5,929 & 56.4 & 198 & 88.8 & \\
\hline Histology & & & & & $<0.001$ \\
\hline Clear cell, angiosarcoma, rhabdomyosarcoma, epithelioid (CARE) histology & 458 & 4.4 & 56 & 25.1 & \\
\hline Synovial & 436 & 4.1 & 10 & 4.5 & \\
\hline Other & 9,614 & 91.5 & 157 & 70.4 & \\
\hline Lymphovascular Invasion & & & & & $<0.001$ \\
\hline Absent & 2,938 & 28.0 & 38 & 17.0 & \\
\hline Present & 199 & 1.9 & 26 & 11.6 & \\
\hline Unknown & 7,371 & 70.1 & 159 & 71.3 & \\
\hline Neurovascular Invasion & & & & & 0.008 \\
\hline Absent & 3,274 & 31.2 & 60 & 26.9 & \\
\hline Present & 113 & 1.1 & 7 & 3.1 & \\
\hline Unknown & 7,121 & 67.8 & 156 & 70.0 & \\
\hline
\end{tabular}




\section{Cureus}

Bone Invasion

$<0.001$

Absent

4,722

$44.9 \quad 117$

54.5

Present

105

$1.0 \quad 10$

4.5

Unknown

5,681

54.196

43.1

\section{TABLE 1: Cohort Characteristics for Trunk/Extremity Soft Tissue Sarcoma Patients With or} Without Lymph Node Metastasis

On univariate analysis (Table 2), older patients and patients with extremity tumors were less likely to have nodal metastasis from STS (OR 0.99, 95\% CI $0.986-0.999, \mathrm{p}=0.043$ and OR $0.59,95 \%$ CI $0.43-0.82, \mathrm{p}=$ 0.002 , respectively). Race and sex were not associated with nodal positivity ( $p>0.1$ ). High grade (Grade 2 vs. Grade 1, OR 6.59, 95\% CI $2.47-17.60$, p < 0.001 and Grade 3 vs. Grade 1, OR 19.03, 95\% CI $7.83-46.29$, p < 0.001), LVI (OR 10.10, 95\% CI $6.01-16.98, \mathrm{p}<0.001$ ), NVI (OR 3.38, 95\% CI $1.51-7.56, \mathrm{p}=0.003$ ), BI (OR $3.84,95 \%$ CI $1.96-7.54, \mathrm{p}<0.001$ ), and tumor size $>5 \mathrm{~cm}$ (OR 3.92, 95\% CI $1.40-10.93, \mathrm{p}=0.009)$ were all associated with LNM. CARE histology was also associated with LNM (CARE histology vs. other, OR 7.49, 95\% CI $5.44-10.3, p<0.001$ ), but the synovial sarcoma histology was not (synovial sarcoma vs. other, OR 1.4, $95 \%$ CI $0.74-2.68, \mathrm{p}=0.666$ ).

\begin{tabular}{|c|c|c|c|c|}
\hline Variable & Univariate Odds Ratio $(95 \% \mathrm{Cl})$ & P-value & Multivariable Odds Ratio $(95 \% \mathrm{Cl})$ & P-value \\
\hline Age (Continuous) & $0.993(0.986-0.999)$ & 0.043 & $0.99(0.987-1.001)$ & 0.087 \\
\hline Sex & & & NA & \\
\hline Male & Reference & & & \\
\hline Female & $1.00(0.77-1.30)$ & 0.999 & & \\
\hline Race & & & NA & \\
\hline White & Reference & & & \\
\hline Black & $1.20(0.81-1.78)$ & 0.355 & & \\
\hline Asian & $1.18(0.55-2.54)$ & 0.668 & & \\
\hline Unknown & $0.49(0.16-1.56)$ & 0.228 & & \\
\hline \multicolumn{5}{|l|}{ Location } \\
\hline Trunk & Reference & & Reference & \\
\hline Extremity & $0.59(0.43-0.82)$ & 0.002 & $0.70(0.50-0.99)$ & 0.042 \\
\hline \multicolumn{5}{|l|}{ Size } \\
\hline$\leq 5 \mathrm{~cm}$ & Reference & & Reference & \\
\hline$>5 \mathrm{~cm}$ & $3.92(1.40-10.93)$ & 0.009 & $4.12(1.42-11.99)$ & 0.009 \\
\hline Unknown & $2.31(1.50-3.55)$ & $<0.001$ & $2.41(1.52-3.80)$ & $<0.001$ \\
\hline \multicolumn{5}{|l|}{ Grade } \\
\hline I & Reference & & Reference & \\
\hline$\|$ & $6.59(2.47-17.60)$ & $<0.001$ & $6.05(2.26-16.23)$ & $<0.001$ \\
\hline III & $19.03(7.83-46.29)$ & $<0.001$ & $15.55(6.36-38.04)$ & $<0.001$ \\
\hline \multicolumn{5}{|l|}{ Histology } \\
\hline Other & Reference & & Reference & \\
\hline CARE & $7.49(5.44-10.30)$ & $<0.001$ & $4.72(3.35-6.66)$ & $<0.001$ \\
\hline Synovial & $1.40(0.74-2.68)$ & 0.303 & $0.98(0.50-1.93)$ & 0.953 \\
\hline npnovascuiar & & & & \\
\hline
\end{tabular}




\section{Cureus}

\begin{tabular}{lllll} 
Absent & Reference & \multicolumn{2}{l}{ Reference } & $<0.001$ \\
Present & $10.10(6.01-16.98)$ & $<0.001$ & $5.86(3.33-10.31)$ & $<0.001$ \\
Unknown & $1.67(1.17-2.38)$ & 0.005 & $2.17(1.43-3.29)$ & 0.751 \\
Neurovascular Invasion & & & & 0.006 \\
Absent & Reference & & Reference & \\
Present & $3.38(1.51-7.56)$ & 0.003 & $1.16(0.47-2.83)$ & 0.006 \\
Unknown & $1.20(0.89-1.61)$ & 0.245 & $1.71(1.17-2.51)$ & $<0.001$ \\
Bone Invasion & & & & \\
Absent & Reference & & Reference & \\
Present & $3.84(1.96-7.54)$ & $<0.001$ & $2.73(1.32-5.61)$ & $0.40(0.28-0.58)$ \\
Unknown & $0.68(0.52-0.90)$ & 0.006 & & \\
\hline
\end{tabular}

TABLE 2: Univariate and Multivariable Logistic Regression Predicting for Lymph Node Metastasis

CARE: clear cell, angiosarcoma, rhabdomyosarcoma, and epithelioid; Cl: confidence interval

Table 2 also shows the multivariable logistic regression analysis. On multivariable analysis, several variables remained associated with LNM. These included Grade 3 tumors (Grade 3 vs. Grade 1, OR 15.55, 95\% CI 6.36 38.04, p < 0.001), LVI (OR 5.86, 95\% CI 3.33 - 10.31, p < 0.001,), BI (OR 2.73, 95\% CI $1.32-5.61, \mathrm{p}=0.006$ ), and tumor size $>5 \mathrm{~cm}(\mathrm{OR} 4.12,95 \% \mathrm{CI} 1.42-11.99, \mathrm{p}=0.009)$. CARE histology was also strongly associated with LNM (OR 4.72, 95\% CI 3.35 - 6.66, p < 0.001), while synovial sarcoma was not (OR 0.98, 95\% CI 0.50 $1.93, \mathrm{p}=0.953)$, and NVI was not associated with LNM on multivariable analysis $(\mathrm{p}>0.1)$.

Adult patients with Grade 3 tumors and CARE histology were identified as a "high-risk" subset. A total of 402 patients in our cohort were designated as high-risk, and this subset of patients had a greater risk of LNM relative to adult patients without both factors: $11.9 \%$ vs. $1.7 \%, \mathrm{p}<0.001$. Furthermore, high-risk patients with either LVI or BI exhibited an extremely high rate of LNM compared to high-risk patients without LVI or BI (33.3\% vs. 9.8\%, $\mathrm{p}<0.001)$ (Figure 2).

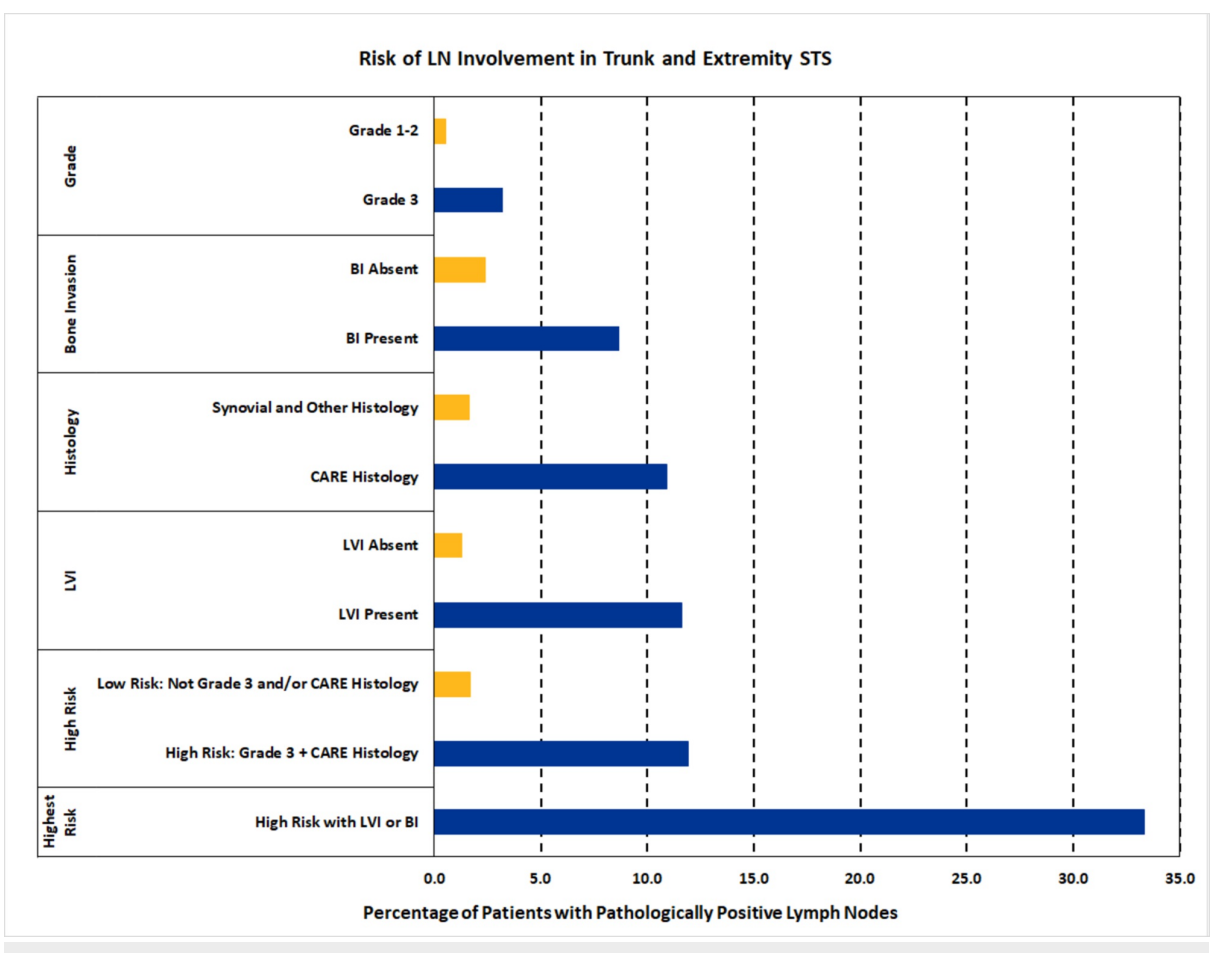

FIGURE 2: Risk of Lymph Node Involvement in Trunk and Extremity Soft 
Tissue Sarcoma (STS)

CARE: clear cell, angiosarcoma, rhabdomyosarcoma, and epithelioid sarcoma; LN: lymph node; LVI: lymphovascular invasion

\section{Discussion}

This is the first large scale study of clinical and histologic predictors of LNM in trunk/extremity STS. LN involvement in non-metastatic STS is associated with high-grade, CARE histology, LVI, and BI. High-risk patients - defined as high-grade with CARE histology - have an 11.9\% risk of LN involvement and can be considered for pathologic LN assessment, particularly if they exhibit LVI or BI, which increased LNM rates to 33\%. Identifying positive lymph nodes in STS may inform prognosis and would likely influence patient management, given the retrospective evidence suggesting a survival benefit for patients with LNM receiving regional lymphadenectomy $[1-3,5-6]$.

The previous studies of LNM in STS primarily evaluated histology and grade as risk factors for nodal involvement $[1,3,7,16-19]$. To our knowledge, the first study of LNM in STS was published in 1978, where a review of over 3,000 cases of STS identified rhabdomyosarcoma and synovial sarcoma to be associated with increased risk of LNM [16]. In 1987, a cohort study of 323 patients showed that high-grade, rhabdomyosarcoma histology, and epithelioid histology were associated with LNM [7]. A larger study of 1,772 patients showed that angiosarcoma, rhabdomyosarcoma, and epithelioid sarcoma had higher rates of LNM [3]. Another study of 1,066 patients confirmed the previously established risk factors and also showed that clear cell sarcoma increased LNM [1].

A more recent publication by Keung et al. using the NCDB showed that lymph node positivity had an impact on survival for non-metastatic patients with certain histologic subtypes of sarcoma [4]. This suggests that pathologic confirmation of nodal disease has a prognostic value, and several retrospective series have shown regional lymphadenectomy to improve survival $[1-3,5,6]$. However, there are no prospective data that show regional lymphadenectomy improves outcomes for patients undergoing surgery for STS. Given the rarity of these tumors and the inherent difficulty of conducting prospective studies, the National Comprehensive Cancer Network (NCCN) has recommended lymphadenectomy for patients with nodal disease based on these retrospective studies. Thus, identifying patients at high-risk for harboring nodal disease is imperative.

This study adds to the current literature by confirming previously identified high-risk histologies and identifying new risk factors for LNM in STS of the trunk/extremity (BI). Furthermore, this study identifies a high-risk cohort with an $11.9 \%$ risk of LNM based on factors that can be determined preoperatively with biopsy alone. This high-risk cohort of patients, particularly those with LVI or BI, may benefit from sentinel node biopsy, and, if positive, should be considered for regional lymphadenectomy based on current NCCN recommendations derived from the aforementioned retrospective studies. Of the 223 pathologically nodepositive patients in our cohort, only 114 patients were coded as clinically node-positive, 47 patients were coded as clinically node-negative, and the remainder did not have a clinical nodal stage recorded. This suggests that at least $21.1 \%$ of the patients (47/223) with nodal disease had no clinical evidence of nodal involvement. It is likely that in some of these cases, nodal metastases were discovered incidentally during resection of a primary tumor, which has been discussed in prior series [2, 4], but it is possible that some of these patients had a sentinel lymph node biopsy (SLNB). Although the role of SLNB in STS has not yet been determined, a prospective study showed that six of 12 patients with clear cell sarcoma had clinically occult LNM identified by SLNB [20], which suggests the technique may be useful in patients at high risk for LNM.

A few limitations should be discussed. As is the case in any retrospective review, we may not have been able to control for unknown variables that may have been unbalanced between the cohorts. Furthermore, many of the variables examined had unknown/missing values, including $5.2 \%$ of tumor size, $70.2 \%$ of LVI, $67.8 \%$ of NVI, and $53.8 \%$ of BI. We excluded patients who received neoadjuvant therapy to avoid treatment effect on LNM; however, this greatly reduced the number of patients included in the study, including patients likely with larger tumors, given only 56 patients in our cohort had a primary tumor $>5 \mathrm{~cm}$. Furthermore, the NCDB does not record data for lymph node size, the presence of extracapsular extension, or the type of procedure conducted for lymph node evaluation. We did not evaluate survival in our cohort since we excluded patients receiving neoadjuvant treatment, which is a group with worse prognostic factors and possibly worse overall survival (OS) [21]. Despite these limitations, this is the largest study of risk factors for LNM in trunk/extremity STS, and the factors identified in this study can be used by practitioners to select patients at high risk for harboring LNM who may benefit from SLNB, even in the absence of clinical evidence of nodal involvement.

\section{Conclusions}

This NCDB study of non-metastatic STS of the trunk and extremity shows that high-grade and CARE histology were associated with pathologically-proven LNM. Adult patients with both features have an overall $11.9 \%$ risk of LNM and may be considered for pathologic LN assessment, particularly with the presence of 
LVI or BI, where the risk of LNM is > 30\%. Patients with proven LNM in the absence of distant metastatic disease can be considered for regional lymphadenectomy based on current NCCN guidelines.

\section{Additional Information \\ Disclosures}

Human subjects: Consent was obtained by all participants in this study. NA (exempt) issued approval NA. The data used in the study were derived from a de-identified NCDB file (2004-2015). The American College of Surgeons and the $\mathrm{CoC}$ have not verified and are neither responsible for the analytic or statistical methodology employed, nor the conclusions drawn from these data by the investigators. As all patient information in the NCDB database is de-identified, this study was exempt from institutional review board evaluation. Animal subjects: All authors have confirmed that this study did not involve animal subjects or tissue. Conflicts of interest: In compliance with the ICMJE uniform disclosure form, all authors declare the following: Payment/services info: All authors have declared that no financial support was received from any organization for the submitted work. Financial relationships: All authors have declared that they have no financial relationships at present or within the previous three years with any organizations that might have an interest in the submitted work. Other relationships: All authors have declared that there are no other relationships or activities that could appear to have influenced the submitted work.

\section{References}

1. Riad S, Griffin AM, Liberman B, et al.: Lymph node metastasis in soft tissue sarcoma in an extremity . Clin Orthop Relat Res. 2004, 426:129-34. 10.1097/01.blo.0000141660.05125.46

2. Behranwala KA, A'Hern R, Omar AM, Thomas JM: Prognosis of lymph node metastasis in soft tissue sarcoma. Ann Surg Oncol. 2004, 11:714-19. 10.1245/ASO.2004.04.027

3. Fong Y, Coit DG, Woodruff JM, Brennan MF: Lymph node metastasis from soft tissue sarcoma in adults. Analysis of data from a prospective database of 1772 sarcoma patients. Ann Surg. 1993, 217:72-77. 10.1097/00000658-199301000-00012

4. Keung EZ, Chiang YJ, Voss RK, et al.: Defining the incidence and clinical significance of lymph node metastasis in soft tissue sarcoma. Eur J Surg Oncol. 2018, 44:170-77. 10.1016/j.ejso.2017.11.014

5. Sawamura C, Matsumoto S, Shimoji T, Ae K, Okawa A: Lymphadenectomy and histologic subtype affect overall survival of soft tissue sarcoma patients with nodal metastases tumor. Clin Orthop Relat Res. 2013, 471:926-31. 10.1007/s11999-012-2568-0

6. Al-Refaie WB, Andtbacka RH, Ensor J, et al.: Lymphadenectomy for isolated lymph node metastasis from extremity soft-tissue sarcomas. Cancer. 2008, p.:1821-26. 10.1002/cncr.23363

7. Mazeron JJ, Suit HD: Lymph nodes as sites of metastases from sarcomas of soft tissue . Cancer. 1987, 60:1800-808. 10.1002/1097-0142(19871015)60:8<1800::aid-cncr2820600822>3.0.co;2-n

8. Daigeler A, Kuhnen C, Moritz R, et al.: Lymph node metastases in soft tissue sarcomas: a single center analysis of 1,597 patients. Langenbecks Arch Surg. 2009, 394:321-29. 10.1007/s00423-008-0371-x

9. Skinner KA, Eilber FR: Soft tissue sarcoma nodal metastases: biologic significance and therapeutic considerations. Surg Oncol Clin N Am. 1996, 5:121-27. 10.1016/S1055-3207(18)30408-3

10. Bilimoria KY, Stewart AK, Winchester DP, Ko CY: The National Cancer Data Base: a powerful initiative to improve cancer care in the United States. Ann Surg Oncol. 2008, 15:683-90. 10.1245/s10434-007-9747-3

11. AJCC Cancer Staging Form Supplement - AJCC Cancer Staging Manual, Eighth Edition . (2018). Accessed: October 30, 2019: http://cancerstaging.org/references-

tools/deskreferences/Documents/AJCC\%20Cancer\%20Staging\%20Form\%20Supplement.pdf.

12. CONSORT 2010. (2010). Accessed: October 30, 2019: http://www.consort-statement.org/consort-2010.

13. Wang $\mathrm{H}$, Miao R, Jacobson A, et al.: Nodal involvement and survival in synovial, clear cell, angio, rhabdo, and epithelioid sarcoma. J Clin Oncol. 2018, 36:11567. 10.1200/JCO.2018.36.15_suppl.11567

14. Jacobs AJ, Morris CD, Levin AS: Synovial sarcoma is not associated with a higher risk of lymph node metastasis compared with other soft tissue sarcomas. Clin Orthop Relat Res. 2018, 476:589-98. 10.1007/s11999.0000000000000057

15. Ariel IM: Incidence of metastases to lymph nodes from soft-tissue sarcomas . Semin Surg Oncol. 1988, 4:2729. 10.1002/ssu.2980040107

16. Weingrad DN, Rosenberg SA: Early lymphatic spread of osteogenic and soft-tissue sarcomas . Surgery. 1978, 84:231-40.

17. Gaakeer HA, Albus-Lutter CE, Gortzak E, Zoetmulder FA: Regional lymph node metastases in patients with soft tissue sarcomas of the extremities, what are the therapeutic consequences?. Eur J Surg Oncol. 1988, 14:151-56.

18. Lee YT, Moore TM, Schwinn CP: Metastasis of sarcomatous lesion in regional lymph node. J Surg Oncol. 1982, 20:53-58. 10.1002/jso.2930200113

19. Ruka W, Emrich LJ, Driscoll DL, Karakousis CP: Prognostic significance of lymph node metastasis and bone, major vessel, or nerve involvement in adults with high-grade soft tissue sarcomas. Cancer. 1988, 62:9991006. 10.1002/1097-0142(19880901)62:5<999::aid-cncr2820620527>3.0.co;2-n

20. Andreou D, Boldt H, Werner M, Hamann C, Pink D, Tunn PU: Sentinel node biopsy in soft tissue sarcoma subtypes with a high propensity for regional lymphatic spread-results of a large prospective trial. Ann Oncol. 2013, 24:1400-405. 10.1093/annonc/mds650

21. Fairweather M, Keung E, Raut CP: Neoadjuvant therapy for soft-tissue sarcomas. Oncology (Williston Park). 2016, 30:99-106. 